\title{
Real-time segmentation for tomographic imaging
}

\author{
Richard Schoonhoven*, Jan-Willem Buurlage*, Daniël M. Pelt*, and Kees Joost Batenburg*† \\ ${ }^{*}$ Computational Imaging Group, Centrum Wiskunde \& Informatica, Amsterdam, Netherlands \\ ${ }^{\dagger}$ Leiden Institute of Advanced Computer Science, Leiden, Netherlands \\ \{richard.schoonhoven, j.buurlage, d.m.pelt, k.j.batenburg\}@ @ cwi.nl
}

\begin{abstract}
In tomography, reconstruction and analysis is often performed once the acquisition has been completed due to the computational cost of the 3D imaging algorithms. In contrast, real-time reconstruction and analysis can avoid costly repetition of experiments and enable optimization of experimental parameters. Recently, it was shown that by reconstructing a subset of arbitrarily oriented slices, real-time quasi-3D reconstruction can be attained. Here, we extend this approach by including realtime segmentation, thereby enabling real-time analysis during the experiment.

We propose to use a convolutional neural network (CNN) to perform real-time image segmentation and introduce an adapted training strategy in order to apply CNNs to arbitrarily oriented slices. We evaluate our method on both simulated and real-world data. The experiments show that our approach enables realtime tomographic segmentation for real-world applications and outperforms standard unsupervised segmentation methods.
\end{abstract}

Index Terms - tomography, machine learning, segmentation

\section{INTRODUCTION}

Tomographic imaging is a widely applicable technique for studying the internal structure of objects using some form of penetrating radiation such as X-rays or an electron beam. Projection images are obtained from a range of angles and a tomographic reconstruction algorithm subsequently computes a 3D image of the internal structure of the object. Currently, reconstruction and analysis are often performed after image acquisition has completed. If processing, reconstruction, and analysis of tomographic data can be run in real time during the experiment, internal dynamic processes of the imaged object can be visualized and analyzed as they occur. Realtime feedback enables online optimization and steering of the imaging setup and experimental conditions which increases the efficiency of experiments and avoids costly repetition.

Despite advances in computationally efficient reconstruction algorithms [1], [2] and in specialized hardware such as Graphic Processing Units (GPUs) [3] and supercomputers [4], full 3D tomographic reconstructions at the rate of data acquisition remain out of reach for most applications. Recently it was shown that real-time reconstruction can be achieved for a small set of randomly oriented 2D slices [5]. These slices can be adjusted on the fly, thereby giving access to a virtual full 3D volume at a fraction of the computational cost. This methodology is called quasi-3D reconstruction, and is implemented in the RECAST3D software package.

Financial support provided by The Netherlands Organisation for Scientific Research (NWO), project numbers 639.073.506 and 016.Veni.192.235. 978$1-7281-6662-9 / 20 / \$ 31.00$ C2020 IEEE

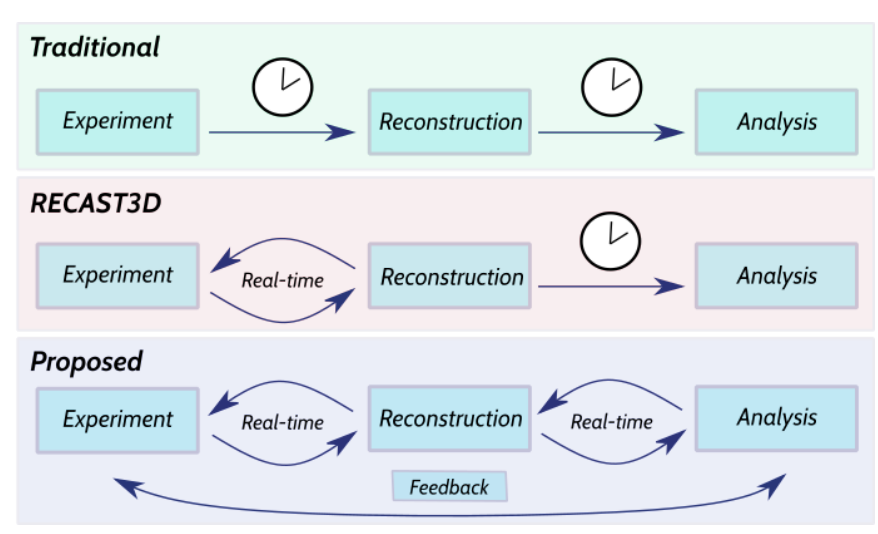

Fig. 1: Traditional experiments (top) involving tomography require significant time for both the reconstruction phase and the offline analysis phase. With RECAST3D (middle) the reconstruction phase is performed in real time. Our method (bottom) additionally includes a real-time segmentation step.

To enable adaptive imaging, where the imaging process is adjusted based on the observations, just having access to a reconstructed volume is not sufficient, as the image analysis step should also be included in the real-time processing pipeline. By real-time we mean that the reconstruction is almost instantly available after data acquisition. In practice, we aim to perform reconstruction and analysis of the quasi3D volume at several frames per second. An important step in many image analysis pipelines is segmentation, which is the problem of assigning to each pixel the appropriate class label from a finite set of classes, for example segmenting bone for calcaneal fractures in CT images [6]. In this article we introduce a real-time imaging pipeline to reconstruct, segment, and visualize quasi-3D volumes implemented as an extension of the existing RECAST3D software package. Our method adds real-time segmentation to the existing real-time reconstruction capabilities of the RECAST3D framework, as outlined in Figure 1.

As quasi-3D reconstruction employs direct reconstruction methods such as filtered backprojection (FBP) [7] and Feldkamp-David-Kress (FDK) [8] without additional image regularization, limited-data artefacts are typically present in the reconstructions. These artifacts limit the applicability of computationally efficient unsupervised segmentation algorithms, such as Otsu's method [9], since they are often unable to separate artifacts and noise from important features. Furthermore, because image analysis algorithms may be sensitive to noise in the segmentation [10], [11], analysis based on 
such traditional segmentation methods may not be accurate. In addition, many unsupervised segmentation methods operate exclusively on the basis of the pixel values [9], [12], [13], limiting their applicability to general segmentation problems as they are unable to segment features that are not based on pixel values.

To overcome these issues, we propose to use a convolutional neural network $(\mathrm{CNN})$ to segment the quasi-3D reconstructions in real time. To apply CNNs in a quasi3D setting, we introduce an adapted training strategy that takes the arbitrary orientations of the slices into account. We show that a CNN is capable of achieving similar accuracy to segmentations based on computationally more expensive total variation minimization (TV-MIN) reconstructions [14] which are too slow to compute for real-time applications. In addition, we show that a CNN can be implemented efficiently as a plugin within the existing RECAST3D framework without significantly increasing the processing time.

This article is structured as follows. In Section II we introduce the tomographic reconstruction problem and define the FDK and TV-MIN reconstruction algorithms. We introduce quasi-3D reconstructions, the segmentation problem, and provide more details on the segmentation plugin. Lastly, we outline our adapted training strategy for randomly oriented slices. In Section III we present the experimental results, and analyze the training strategies. We perform a real-world experiment on a dynamic X-ray CT dataset and two simulated experiments. Finally, in Section IV we state our final conclusions.

\section{METHOD}

\section{A. Prerequisites}

Tomographic Reconstruction: The tomographic reconstruction problem is to recover a volume from a series of its projections. In this article we consider circular cone-beam tomography, where the object is placed in between a point source and flat-panel detector which are situated on opposite sides of a circle. The object is rotated and X-ray projections are taken at a selection of equidistant angles. The approach generalizes to other acquisition geometries (e.g. parallel beam) in a straightforward manner.

The tomographic reconstruction problem can be modelled as an inverse problem:

$$
K \mathbf{u}=\mathbf{f} .
$$

Here $K$ is the forward projection operator, $\mathbf{u} \in \mathbb{R}^{N_{x} \times N_{y} \times N_{z}}$ represents the object, and $\mathbf{f} \in \mathbb{R}^{N_{\theta} \times N_{a} \times N_{b}}$ is the measured projection data, with $N_{\theta}$ is the number of projection angles, and $N_{a}, N_{b}$ are the number of detector rows and columns respectively. In this article we use the FDK reconstruction algorithm, given by

$$
\mathbf{u}_{\mathrm{FDK}}=K^{T}(\mathbf{h} * \tilde{\mathbf{f}}) .
$$

Here $\tilde{\mathbf{f}}$ denotes weighted projection data, which compensates for diminishing intensity at distance from detector center, and $\mathbf{h} \in \mathbb{R}^{N_{b}}$ is a $1 \mathrm{D}$ filter. We used the Ram-Lak filter for this work.
Instead of using FDK, equation 1 can be solved by iteratively minimizing $\|K \mathbf{u}-\mathbf{f}\|$. In addition, we can add prior information about the gradient of the image being sparse by adding a total variation term [14] to improve reconstruction accuracy when projection data is limited or noisy:

$$
\frac{1}{2}\|K \mathbf{u}-\mathbf{f}\|_{2}^{2}+\lambda\|\nabla \mathbf{u}\|_{1}
$$

This function can be minimized by a range of convex optimization algorithms.

Quasi-3D Reconstruction: Quasi-3D reconstruction has recently been proposed as a method to make real-time tomographic reconstruction feasible [5]. Instead of computing a full 3D volume, only a small collection of arbitrarily oriented $2 \mathrm{D}$ slices is reconstructed and visualized in real-time. When these slices are translated and/or rotated by the user, they are reconstructed on the fly, so that it appears as though a full 3D reconstruction is available. This on-demand $2 \mathrm{D}$ reconstruction significantly reduces the total computational cost compared to full 3D reconstruction. This approach is implemented in the open source RECAST3D software package and more implementation details can be found in [5].

In RECAST3D, the filtering and weighting steps of the FDK algorithm are performed in parallel. The computation of $\mathbf{h} * \tilde{\mathbf{f}}$ is performed in real time from the incoming data. When a slice is requested, the application of $K^{T}$ (called backprojection) is performed using GPU-based high-performance routines from the ASTRA toolbox [15]. In addition, a low-resolution 3D FDK reconstruction is created so that the user can preview the object. Our quasi-3D pipeline for segmentation is implemented by extending the RECAST3D software package with a computationally efficient segmentation plugin.

Segmentation: Mathematically, segmenting an image can be described as finding a function $g: \mathbb{R}^{m \times n} \rightarrow \mathbb{Z}_{k}^{m \times n}$, where $m, n$ are the rows and columns of the image and $k$ is the number of object classes to be assigned.

Classical segmentation methods (for example local and global thresholding [9], [12], watershed methods [13]) typically operate on the image greyvalues to separate classes and have the high computational efficiency that is need for realtime segmentation. As an example, Otsu's method performs a segmentation of an image by selecting a threshold that minimizes intra-class variance. In addition to the greyscale distribution, segmentation can be performed on other properties by for example clustering pixels [16] or defining edge boundaries in the image [17]. Recently, CNNs have proven successful for image segmentation [18], [19].

CNNs for segmentation: In this work we use CNNs to segment the tomographic reconstructions. In a segmentation network, the final output layer will assign one of $k$ classes to each pixel. The $\mathrm{CNN}$ is defined by its architecture with weights $\Theta$ which can be altered to change the output. For a given $\Theta$, a CNN corresponds to a function $F_{\Theta}: \mathbb{R}^{m \times n} \rightarrow \mathbb{R}^{k \times m \times n}$ which aims to approximate $g$ by computing a probability vector with predictions for each class for each pixel. The 


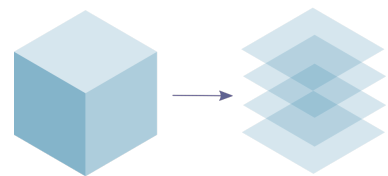

Unidirectional

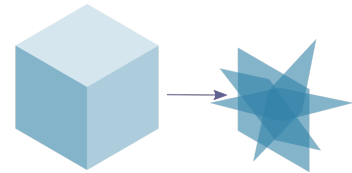

Omnidirectional

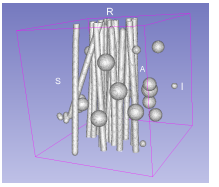

(a)

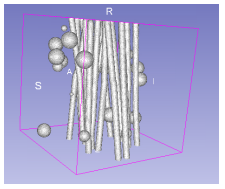

(b)

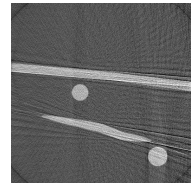

(c)

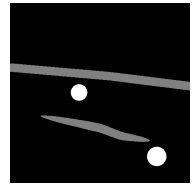

(d)
Fig. 2: Diagram outlining unidirectional training on slices (left) and omnidirectional training (right)

highest probability class can be chosen from the network predictions to obtain a final segmentation.

The weights $\Theta$ are found in a training phase, where input samples $\mathbf{x}_{1}, \ldots, \mathbf{x}_{N}$ are processed by the network and compared to known labelled output samples $\mathbf{y}_{1}, \ldots, \mathbf{y}_{N}$. A loss function $\mathcal{J}: \mathbb{R}^{k \times m \times n} \times \mathbb{Z}_{k}^{m \times n} \rightarrow \mathbb{R}$, such as cross-entropy loss, measures the error of the network on the training samples. The aim of the training phase is to find a $\Theta$ that minimizes the loss on the training dataset

$$
\Theta^{*}=\underset{\Theta}{\arg \min }\left\{\sum_{i=1}^{N} \mathcal{J}\left(F_{\Theta}\left(\mathbf{x}_{i}\right), \mathbf{y}_{i}\right)\right\} .
$$

For a $\mathrm{CNN}$, we can compute the partial derivatives of $\mathcal{J}$ with respect to the weights using backpropagation. The weights can be updated using gradient-based optimization algorithms [20].

\section{B. Quasi-3D training strategies}

In 3D image segmentation, neural networks are often trained on $2 \mathrm{D}$ slices from the volumes since full 3D networks are typically computationally too expensive [21], [22]. The 2D input slices are obtained by extracting slices in a single direction. In contrast, slices in a quasi-3D reconstruction can have an arbitrary orientation. A network trained only on unidirectional slices may not recognize object classes from a different view. Therefore, the standard training procedure has to be adapted to enable application of CNNs to arbitrary oriented slices. Here, we introduce a training strategy where arbitrarily oriented $2 \mathrm{D}$ slices of the tomographic volume are supplied as input for the neural network.

Let $\mathbf{X} \in \mathbb{R}^{n \times n \times n}$ be an input volume and $\mathbf{Y} \in \mathbb{Z}_{k}^{n \times n \times n}$ the aligned target volume. Define $E_{\alpha, \beta, \gamma}: \mathbb{Z} \times \mathbb{R}^{n \times n \times n} \rightarrow$ $\mathbb{R}^{n \times n}$ to be a rotated extraction operation. $E_{\alpha, \beta, \gamma}(i, \mathbf{X})$ extracts the $i$-the slice rotated by angles $\alpha, \beta, \gamma$ with respect to the sagittal slice from the volume $\mathbf{X}$. For omnidirectional training we create a dataset of slices with pairs $\left(E_{\alpha, \beta, \gamma}(i, \mathbf{X}), E_{\alpha, \beta, \gamma}(i, \mathbf{Y})\right)$ where the angles are randomly generated. For unidirectional training we create pairs of sagittal slices $\left(E_{0,0,0}(i, \mathbf{X}), E_{0,0,0}(i, \mathbf{Y})\right)$ (see Figure 2).

\section{Segmentation Plugin}

To construct the pipeline for real-time segmentation we developed a plugin for RECAST3D which segments quasi$3 \mathrm{D}$ reconstructions. The segmented slices are then visualized in RECAST3D. The plugin is GPU-based and can be disabled, altered and reenabled while the projection data is processed simultaneously. A command line interface allows for online tuning of parameters with immediate visual feedback, and the
Fig. 3: (a), (b) Example volumes of the fibre-sphere data, (c) slice of the noisy FDK reconstruction, and (d) slice of the ground truth (spheres and fibres have different labels).

user can select which class is visualized. The plugin operates independently from the quasi-3D reconstruction pipeline, and can be run on a separate node.

The plugin is implemented in Python, and includes three CNNs implemented in PyTorch: the MS-D network [22], [23], U-Net [18], and ResNet [24]. In addition, the plugin includes several traditional segmentation methods, including Otsu's method [9], cross entropy thresholding [12], contour evolution [25], region based random walk segmentation [26] and the watershed algorithm [13].

\section{RESULTS AND DISCUSSION}

\section{A. Setup}

To assess the accuracy and computational efficiency of our CNN-based segmentation approach, we compare it with traditional unsupervised methods. The neural network used in this work is the MS-D network [22], chosen because of its low number of trainable parameters.

Since the MS-D network can flexibly adapt to different problems, we used the same network architecture for each experiment. We used an MS-D network, implemented in PyTorch [23], of 100 layers with a width of 1 . The dilations in layer $i$ were set to $1+(i \bmod 10)$. The networks were trained using the ADAM algorithm [20], using a batch size of 20, and the cross-entropy loss function. For each experiment, the data was split in training, validation, and test sets. The network is trained on the training set for 100 epochs. The network with the lowest validation error was selected to be evaluated on the test set. All experiments were run on a workstation with an AMD Ryzen 3800X processor and NVIDIA GeForce RTX 2070 Super GPU. To quantify our comparisons we use the F1-score (also known as the Dice coefficient), and the accuracy. For multi-class problems we report the macro F1score (average of per-class F1-score), and the global accuracy.

\section{B. Simulated data}

We created two sets of simulated tomographic data to investigate the difference between omni- and unidirectional training, and to quantitatively compare Otsu's method to the MS-D network. For the former we created twenty $512^{3}$ volumes filled with fibre strands and spheres. Each volume contained 20 spheres and 20 fibres which were generated with a random shape and location. Greyscale intensities were the same for both classes. 3D renderings of the noiseless volumes are shown in Figure 3.

For the second simulation experiment we created twenty $512^{3}$ volumes filled with 40 fibre strands generated with 


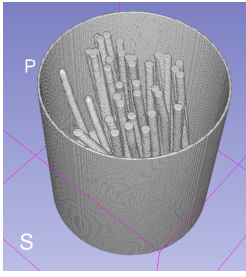

(a)

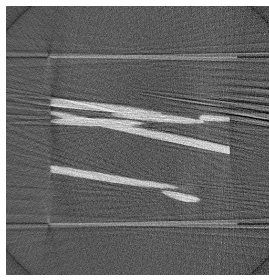

(b)

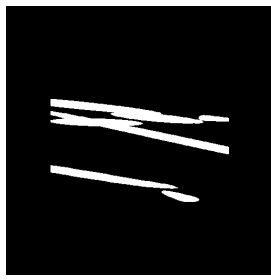

(c)
Fig. 4: (a) Example volume of the fibre-container data with container, (b) slice of the noisy FDK reconstruction, and (c) slice of the ground truth (the container is not to be segmented).

\begin{tabular}{l|ll|ll} 
& \multicolumn{2}{|c|}{ Random orientation } & \multicolumn{2}{c}{ Sagittal orientation } \\
Training & F1 & Accuracy & F1 & Accuracy \\
\hline Omnidirectional & $\mathbf{0 . 9 9 8 9}$ & $\mathbf{0 . 9 9 7 9}$ & 0.9951 & 0.9950 \\
Unidirectional & 0.6857 & 0.9792 & $\mathbf{0 . 9 9 9 5}$ & $\mathbf{0 . 9 9 9 5}$
\end{tabular}

TABLE I: Macro F1 and accuracy on the fibre-sphere (left) randomly oriented test set, and (right) sagittal test set, for MS-D networks trained with the omnidirectional, and unidirectional strategies.

random shapes surrounded by a cylinder forming a container (see Figure 4). Greyscale intensities were the same for the container and the fibres to represent a segmentation problem where the fibres are to be labeled but the container is not.

Using the ASTRA toolbox [15] we simulated a cone-beam projection dataset of 60 projections for each volume. Furthermore, Poisson noise was applied to the projection dataset where the sample absorbed roughly $70 \%$ of the incoming photons. Out of the 20 phantoms, 14 were randomly selected for training, 4 for validation and 2 for testing. For each scan we obtained 500 randomly oriented, and 500 unidirectional $512 \times 512$ slices for both the ground truth labeled volume, and the noisy FDK reconstruction (see Figures 3 and 4).

Comparison of uni- and omnidirectional training slices:

To investigate the importance of the slicing direction in the training strategy for CNNs, we compare unidirectional and omnidirectional training strategies. We trained one network on the slices in a single direction and the other on randomly oriented slices, using the fibre-spheres dataset (Figure 3). Otsu's method, and any other unsupervised method that works solely on greyvalues, are not applicable to this type of multiclass segmentation problem. This indicates an advantage of deep-learning approaches.

Example results from the test set are shown in Figure 5. Note that both networks were able to remove the Poisson noise and the tomographic artifacts. Some notable differences between both networks can be seen where the unidirectional network identifies parts of the fibre strands as spheres. In Table I we report the macro F1-score, and accuracy for both networks on both the test set with randomly oriented slices and the test set with slices in a single direction.

The MS-D omnidirectional network outperforms the unidirectional network on both quantitative measures for the randomly oriented test set, which is in line with the qualitative comparison shown in Figure 5. When tested on the sagittal direction, although the unidirectional networks performs better than the omnidirectional network, the difference in perfor-
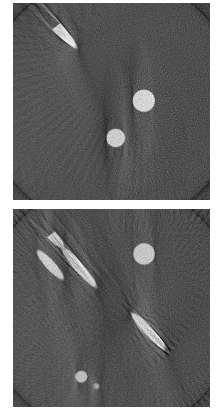

FDK
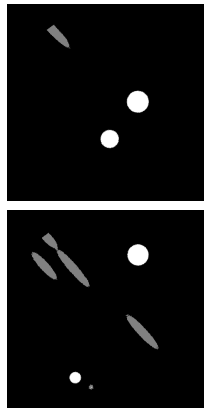

ground truth
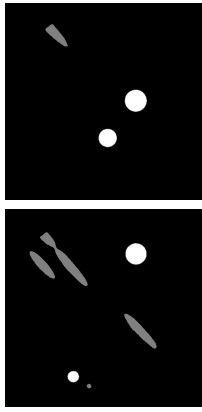

MS-D omnidirectional
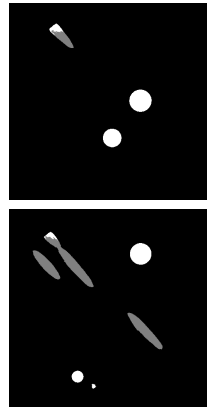

MS-D unidirectional
Fig. 5: Slices of the simulated fibre-sphere test dataset and MS-D network predictions From left to right we have the FDK reconstructions, the labeled ground truths, the MSD network output trained on randomly oriented slices, and the MS-D network output trained on unidirectional slices.

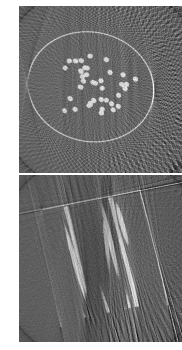

FDK

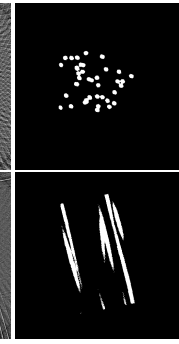

ground truth

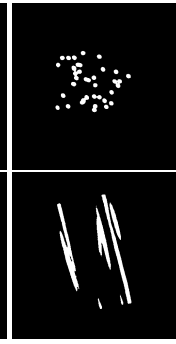

MS-D omnidi- MS-D rectional

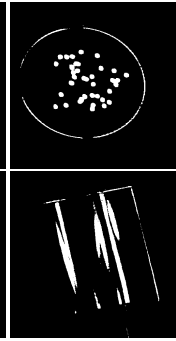

unidirectional

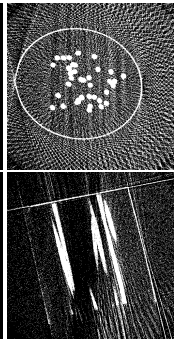

Otsu
Fig. 6: Slices of the simulated fibre-container test dataset, with (from left to right) the FDK reconstructions, the labeled ground truths, the omnidirectional MS-D network output, the unidirectional MS-D network output, and Otsu's method.

mance is significantly smaller. This can be explained by the fact that the omnidirectional network has also encountered images sliced in the sagittal direction.

\section{Comparison of MS-D segmentation and Otsu's method:}

Here, we compare Otsu's method to the uni- and omnidirectional MS-D networks. Both the MS-D networks and Otsu's method were tested on randomly oriented slices from the fibre data test phantoms because the user can select arbitrary slices in RECAST3D. To more accurately determine the performance of Otsu's method inside the container, we created a version of Otsu's method where a ROI-mask was applied on the segmented slices with the proper rotation. We used a cylindrical volume around the simulated container to remove misclassified background. Some example results from the test set can be seen in Figure 6 and in Table II we report the F1-score and accuracy.

The results show that the omnidirectional MS-D network was able to accurately segment the fibres and remove the applied Poisson noise. We see that the unidirectional MS-D network misclassified the container in the randomly oriented slices, and that Otsu's method occasionally does not remove the FDK artifacts and noise. In addition, Otsu's method classifies the container as a fibre since it is unable to distinguish it from the fibres on the basis of intensity. Even if we manually mask the region-of-interest, the interior of the container is 


\begin{tabular}{l|ll} 
& F1-score & Accuracy \\
\hline MS-D omnidirectional & $\mathbf{0 . 9 5 4 4}$ & $\mathbf{0 . 9 9 8 9}$ \\
MS-D unidirectional & 0.6777 & 0.9885 \\
Otsu & 0.0585 & 0.6086 \\
ROI-Otsu & 0.2568 & 0.9300
\end{tabular}

TABLE II: F1 and accuracy on the fibre-container randomly oriented test set for MS-D omnidirectional, MS-D unidirectional, Otsu, and ROI Otsu.

significantly more noisy than the MS-D network segmentation.

The MS-D network outperforms Otsu and ROI-Otsu on both metrics. Otsu's method performs significantly worse on F1score, which can be explained by the greater amount of false positives segmented by Otsu as opposed to the MS-D network.

\section{Experimental data}

To show the feasibility of our method in real-world applications, we applied the real-time segmentation pipeline to a real-world dynamic X-ray CT dataset of a dissolving tablet suspended in gel [27], [28]. A container with a dissolving tablet was filled with gel to create moving air bubbles which we segmented. The container was rotated at $100 \mathrm{deg} / \mathrm{s}$ and 60 projections were acquired every 180 degrees with an exposure time of $30 \mathrm{~ms}$ for each projection. In total 9960 projections of size $647 \times 768$ were taken and the experiment lasted 5 minutes.

In RECAST3D, the full processing step of a batch of projections takes approximately $140 \mathrm{~ms}$ on our workstation. The computation time to compute the backprojection for a slice is about 2 milliseconds. The segmentation with Otsu's method is about 3 milliseconds and with the MS-D network about 30 milliseconds. This means that the pipeline would be able to dynamically visualize the projection data stream every 170 milliseconds for a batch of 60 projections. In this experiment, data acquisition was at a rate of 1.8 seconds per batch of 60 projections (180 degrees), well within the computational limits of the pipeline. Figure 8 shows an example real-time quasi-3D reconstruction and segmentation of the data in RECAST3D.

To create training data for the neural networks we created TV-MIN reconstructions for every 60 projections with a regularization parameter $\lambda=0.001$ for 2000 iterations. We used the Douglas-Rachford primal-dual splitting algorithm [29] to iteratively minimize the functional. Each TV-MIN reconstruction took roughly 20 hours on our workstation and is therefore infeasible to compute in real time. Next, we created 25 labeled ground truth volumes by applying Otsu's method to the TV-MIN reconstructions and masking the region outside the container. As a final processing step we removed small objects with a mass smaller than 4 pixels with the scikit-image remove_small_objects function from the morphology package [30]. The scans were randomly separated into 18 training scans (9216 slices), 4 validation scans (2048 slices) and 3 test scans (1536 slices) for the unidirectional network. For the arbitrarily oriented slices, we chose the same amount of slices at random 3D orientations for each scan.

To compare our method to an existing computationally efficient method, we segmented each FDK slice with Otsu's method and manually applied a cylindrical ROI-mask to remove misclassified background. The results can be seen in

\begin{tabular}{l|ll} 
& F1-score & Accuracy \\
\hline MS-D omnidirectional & $\mathbf{0 . 8 8 1 6}$ & $\mathbf{0 . 9 9 8 3}$ \\
MS-D unidirectional & 0.7595 & 0.9968 \\
Otsu & 0.0142 & 0.2538 \\
ROI-Otsu & 0.8229 & 0.9977
\end{tabular}

TABLE III: F1 and accuracy on the real-world TabletInFluid randomly oriented test set for MS-D omnidirectional, MS-D unidirectional, Otsu, and ROI Otsu.

Figure 7. In Table III we report the F1-score and accuracy for the randomly oriented slices.

The MS-D network trained on randomly rotated slices is able to create an accurate segmentation of the bubbles in real time and it outperformed the other three methods on all metrics. It is able to create real-time segmentations with similar quality to the computationally expensive segmented TV-MIN reconstructions. This shows that our method can be used to perform quasi-3D reconstruction and segmentation in real time by training on randomly oriented slices of segmented TV-MIN reconstructions. Note that, in practice, acquiring training data and training the networks has to be performed offline. The results show that our method outperforms Otsu's method with masking on all metrics. Notably, the unidirectional network regularly misclassifies sections of the randomly oriented slices. The importance of the training method is highlighted when comparing the F1-scores for both MS-D networks.

\section{CONClusions}

In this paper, we introduced a real-time pipeline to process, reconstruct, and segment quasi-3D tomographic images, representing an important step for online and real-time analysis of tomographic experiments. We showed the importance of including arbitrarily oriented slices in the training dataset to achieve accurate results. We demonstrated that a deep-learning based approach can perform better than Otsu's method in terms of accuracy on both simulated data and real-world dynamic tomographic data. In addition, our deep-learning based approach is more generalizable to multi-class segmentation problems than traditional intensity-based unsupervised segmentation methods. Using our method, one can perform realtime and online segmentation of quasi-3D volumes, enabling immediate feedback and analysis during experiments.

\section{REFERENCES}

[1] S. Basu and Y. Bresler, " $O\left(N^{3} \log N\right)$ backprojection algorithm for the 3-D Radon transform," IEEE Transactions on Medical Imaging, vol. 21, pp. 76-88, 2002.

[2] D. Kim, S. Ramani, and J. A. Fessler, "Combining Ordered Subsets and Momentum for Accelerated X-Ray CT Image Reconstruction," IEEE Transactions on Medical Imaging, vol. 34, no. 1, pp. 167-178, 2015.

[3] W. Palenstijn, K. Batenburg, and J. Sijbers, "Performance improvements for iterative electron tomography reconstruction using graphics processing units (GPUs)," Journal of structural biology, vol. 176, no. 2, pp. 250-253, 2011.

[4] T. Bicer, D. Gürsoy, R. Kettimuthu, F. D. Carlo, G. Agrawal, and I. T. Foster, "Rapid tomographic image reconstruction via large-scale parallelization," in Euro-Par, 2015.

[5] J.-W. Buurlage, H. Kohr, W. J. Palenstijn, and K. J. Batenburg, "Realtime quasi-3D tomographic reconstruction," Measurement Science and Technology, vol. 29, no. 6, p. 064005, Apr 2018. 


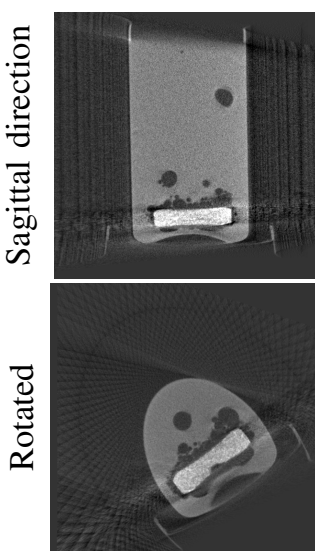

FDK

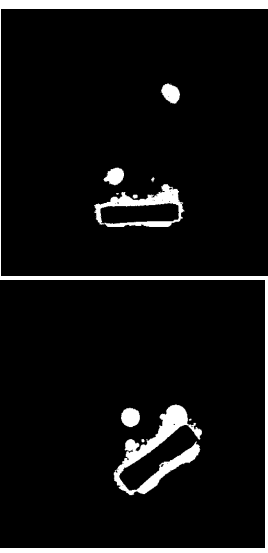

TV-MIN ground truth

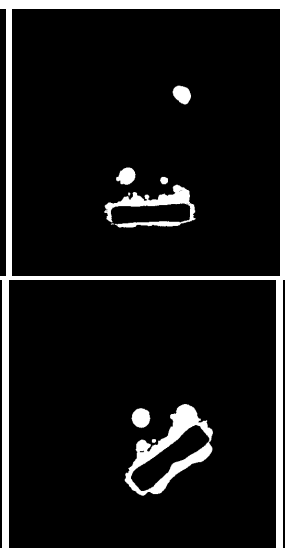

MS-D omnidirectional

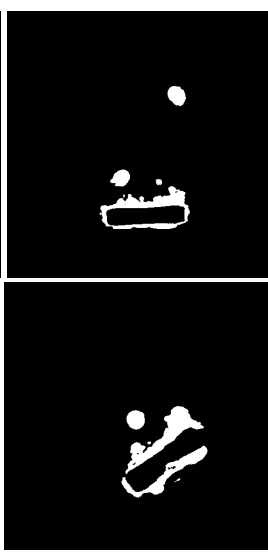

MS-D unidirectional

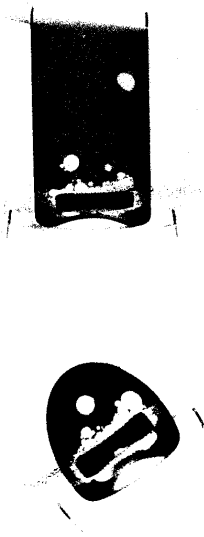

Otsu

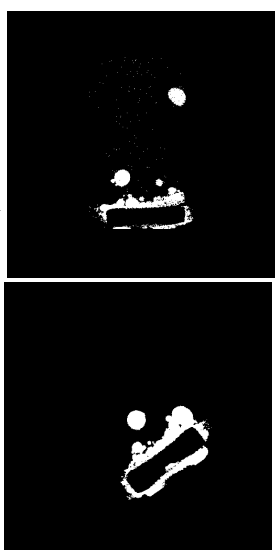

ROI-Otsu

Fig. 7: Slices of the TabletInFluid test dataset and experimental predictions. The first row is from the unidirectional dataset, the second from the randomly rotated dataset. From left to right we have the FDK reconstructions, the labeled TV-MIN ground truths, the MS-D network output trained on randomly oriented slices, the MS-D network output trained on slices in one direction, Otsu's segmentation, and Otsu's segmentation using a ROI cylindrical mask.
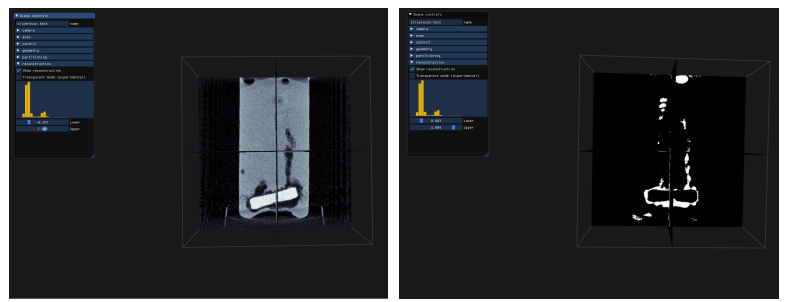

Fig. 8: (Left) example screenshot of dissolving tablet data reconstructed dynamically in RECAST3D, (right) example of segmented network output in RECAST3D.

[6] W. Rahmaniar and W.-J. Wang, "Real-Time automated segmentation and classification of calcaneal fractures in CT images," Applied Sciences, vol. 9, no. 15, p. 3011, 2019.

[7] A. C. Kak and M. Slaney, Principles of Computerized Tomographic Imaging. Society for Industrial and Applied Mathematics, 2001.

[8] L. A. Feldkamp, L. C. Davis, and J. W. Kress, "Practical cone-beam algorithm," J. Opt. Soc. Am. A, vol. 1, no. 6, pp. 612-619, Jun 1984.

[9] N. Otsu, "A Threshold Selection Method from Gray-Level Histograms," IEEE Transactions on Systems, Man, and Cybernetics, vol. 9, no. 1, pp. 62-66, Jan 1979.

[10] Y. Mingqiang, K. Kidiyo, and R. Joseph, "A survey of shape feature extraction techniques," Pattern recognition, vol. 15, no. 7, pp. 43-90, 2008.

[11] A. Rodriguez, H. Zhang, K. Wiklund, T. Brodin, J. Klaminder, P. Andersson, and M. Andersson, "Refining particle positions using circular symmetry," PLOS ONE, vol. 12, no. 4, pp. 1-23, 042017.

[12] C. Li and P. K.-S. Tam, "An iterative algorithm for minimum cross entropy thresholding," Pattern Recognition Letters, vol. 19, pp. 771776, 1998.

[13] P. Neubert and P. Protzel, "Compact Watershed and Preemptive SLIC: On Improving Trade-offs of Superpixel Segmentation Algorithms," in 2014 22nd International Conference on Pattern Recognition, Aug 2014, pp. $996-1001$.

[14] A. Beck and M. Teboulle, "Fast Gradient-Based Algorithms for Constrained Total Variation Image Denoising and Deblurring Problems," IEEE Transactions on Image Processing, vol. 18, no. 11, pp. 24192434, Nov 2009.

[15] W. van Aarle, W. J. Palenstijn, J. Cant, E. Janssens, F. Bleichrodt, A. Dabravolski, J. D. Beenhouwer, K. J. Batenburg, and J. Sijbers, "Fast and flexible X-ray tomography using the ASTRA toolbox," Opt. Express, vol. 24, no. 22, pp. 25 129-25 147, Oct 2016.

[16] N. Dhanachandra, K. Manglem, and Y. J. Chanu, "Image segmentation using K-means clustering algorithm and subtractive clustering algo- rithm," Procedia Computer Science, vol. 54, pp. 764-771, 2015.

[17] R. Muthukrishnan and M. Radha, "Edge detection techniques for image segmentation," International Journal of Computer Science \& Information Technology, vol. 3, no. 6, p. 259, 2011.

[18] O. Ronneberger, P. Fischer, and T. Brox, "U-net: Convolutional networks for biomedical image segmentation," in International Conference on Medical image computing and computer-assisted intervention. Springer, 2015, pp. 234-241.

[19] V. Badrinarayanan, A. Kendall, and R. Cipolla, "Segnet: A deep convolutional encoder-decoder architecture for image segmentation," IEEE transactions on pattern analysis and machine intelligence, vol. 39, no. 12, pp. 2481-2495, 2017.

[20] D. Kingma and J. Ba, "Adam: A Method for Stochastic Optimization," International Conference on Learning Representations, 122014.

[21] G. Litjens, T. Kooi, B. E. Bejnordi, A. A. A. Setio, F. Ciompi, M. Ghafoorian, J. A. Van Der Laak, B. Van Ginneken, and C. I. Sánchez, "A survey on deep learning in medical image analysis," Medical image analysis, vol. 42, pp. 60-88, 2017.

[22] D. M. Pelt and J. A. Sethian, "A mixed-scale dense convolutional neural network for image analysis," Proceedings of the National Academy of Sciences, vol. 115, no. 2, pp. 254-259, 2018.

[23] A. A. Hendriksen, "Mixed-scale Dense Networks for PyTorch," https: //github.com/ahendriksen/msd_pytorch, 2020.

[24] K. He, X. Zhang, S. Ren, and J. Sun, "Deep Residual Learning for Image Recognition," in 2016 IEEE Conference on Computer Vision and Pattern Recognition (CVPR), 2016, pp. 770-778.

[25] P. Márquez-Neila, L. Baumela, and L. Alvarez, "A Morphological Approach to Curvature-Based Evolution of Curves and Surfaces," IEEE Transactions on Pattern Analysis and Machine Intelligence, vol. 36, no. 1, pp. 2-17, Jan 2014.

[26] L. Grady, "Random Walks for Image Segmentation," IEEE Transactions on Pattern Analysis and Machine Intelligence, vol. 28, no. 11, pp. 17681783, Nov 2006.

[27] S. B. Coban and F. Lucka, "Dynamic 3D X-ray micro-CT data of a tablet dissolution in a water-based gel," Oct. 2019. [Online]. Available: https://doi.org/10.5281/zenodo.3610187

[28] S. B. Coban, F. Lucka, W. J. Palenstijn, D. Van Loo, and K. J. Batenburg, "Explorative Imaging and Its Implementation at the FleX-ray Laboratory," Journal of Imaging, vol. 6, no. 4, p. 18, 2020. [Online]. Available: "https://doi.org/10.3390/jimaging6040018",

[29] R. Boţ and C. Hendrich, "A Douglas-Rachford type primal-dual method for solving inclusions with mixtures of composite and parallel-sum type monotone operators," SIAM Journal on Optimization, vol. 24, pp. 25412565, 012013.

[30] S. van der Walt, J. L. Schönberger, J. Nunez-Iglesias, F. Boulogne, J. D. Warner, N. Yager, E. Gouillart, T. Yu, and the scikit-image contributors, "Scikit-image: image processing in Python," PeerJ, vol. 2, p. e453, 6 2014. 